\title{
Physical Properties of Energy-Momentum Tensor of Gravitational Field in RAF Theory
}

\author{
Branko M. Novakovic
}

\begin{abstract}
In Relativistic Alpha Field Theory (RAFT or RAF theory) there are two the most intriguing conclusions. The first one is that the Energy Momentum Tensor (EMT) of gravitational static field obeys the following properties: 1) the symmetry, 2) the non-negative energy density and 3) zero trace of EMT. Here the non-negative energy density means that the energy density is positive, but at the radius $r_{c}=G M / c^{2}$ is zero. This radius corresponds to the point where the acceleration is equal to zero because negative acceleration is changing into the positive one. This is the crucial difference between RAF theory of gravitational static field and field gravity approach, where energy density is positive and without null point. At the radius $r_{c}$ free fall velocity is maximal and equal to the speed of the light in vacuum $c$. The second conclusion is that the gravity force is attractive (in the region $r_{c}<r<\infty$ ) and repulsive (in the region $r_{\min } \leq r<r_{c}$, where $\left.r_{\text {min }}=G M / 2 c^{2}\right)$. At the minimal radius $r_{\text {min }}$, repulsive force is maximal.
\end{abstract}

Index terms - Relativistic Alpha Field Theory (RAFT), Physical properties of EMT in a gravitational static field, Attractive gravitational force, Repulsive gravitational force, Extremely strong gravitational fields.

\section{INTRODUCTION}

As it is well known, the problem of mathematical and physical definition of energy-momentum relation of the gravitational field started with the introduction of General Relativity (GR) [1-5]. Namely, GR is based on the geometrical approach to the description of a gravitational field. The most important feature of the Einstein's equations is that the right side of the equations does not include energy-momentum of the gravitational field itself. This corresponds to the conclusion that in GR theory the gravity is not a material field. As the consequence, the Einstein's equations do not express the energy conservation of matter plus gravitational field. Further consequences are negative energy density for a static gravitational field and violation of the energy conservation in expanding space-time. The absence of the energy density of the gravitational field itself in GR also leads to the problem of quantization of the gravitational field, because one cannot define the energy of graviton as quanta of the field.

There are several attempts in combination of GR and field approach in field gravity theories [6-8] in order to solve the mentioned problems. But, in the reference [9], it has been shown that there is the internal inconsistency in the mentioned theories. The common disadvantage of the mentioned approaches is the fact that they do not satisfy the all of the required physical properties of the energy-momentum tensor (EMT) for gravitational static field. Namely, from the quantum relativistic field theories of the

Branko Novakovic, FSB - University of Zagreb, Luciceva 5, P.O.B. 509, 10000 Zagreb, Croatia other physical interaction [10], the EMT of massless boson field obeys the following three important conditions: symmetry, $T_{\mu \eta}=T_{\eta \mu}$; positive energy density for static and free field, $T_{00}>0$; and zero trace, $T=0$. These conditions must be fulfilled within the consistent field approach for both static and free fields, as it is the case of the electromagnetic field.

The field approach to gravity was also considered in a number of studies [11-13]. Quantum description of the field approach is presented in the references [14-17]. The basic principles of the field gravity theory have been discussed in the reference [18]. A consistent field gravity theory, based on inertial frames, Minkowski space-time and localizable positive energy of the gravitational field, has been discussed in [19-22]. The common physical elements in the description of all fundamental interaction are pointed out in the references [10,18,21]. Generally, there are two the most intriguing consequences of the consistent field gravity theory [10]. The first one is that the EMT obeys the required properties: 1) the symmetry, 2) the positive energy density for static and free field and 3) zero trace of massless field. The second consequence is that the gravity force has an ordinary quantum nature and is presented by the sum of the attraction (spin 2) and repulsion (spin 0) components.

In this paper we use the geometrical approach in the Relativistic Alpha Field Theory (RAFT) of gravitational static field [23-25]. The basic difference between RAF theory and classical General Relativity (GR) $[2,26-30]$ is in the process of the determination of the field parameters. Namely, in RAF theory two dimensionless scalar field parameters $\alpha$ and $\alpha^{\prime}$ are introduced [23]. These parameters are determined on the condition that they must satisfy both the covariant energy equation and the geometric properties of the Einstein's field equations. On that way, the field parameters $\alpha$ and $\alpha^{\prime}$ become the functions of the normalized potential energy of a particle in a gravitational field. As the consequence, the EMT on the right side of the field equations is automatically derived from the left side of the same equations. Further, RAF theory solves the following items: problem of singularity by discovery of the existence of the minimal radius in gravitational field [24], problem of determination of the source of dark energy by discovery of a positive (repulsive) gravitational force $[25,33]$ and problem of unification of the other fundamental forces with gravitational force in the standard four dimensions (4D) [23-25]. In the recent papers it has been shown that the singularity and dark energy in GR are direct consequences of vacuum solution of field equations [31] and that RAF theory extends the applications of GR to the extremely strong gravitational field, including Planck's scale [32]. Thus, RAF theory is general field theory in the sense that it can be applied 
both to weak and extremely strong gravitational field. In the case of the relatively weak field (like in our solar system) RAF theory is reduced to GR. In the case of the total vacuum without any potential field $\left(\alpha=\alpha^{\prime}=1\right)$, RAF theory is reduced to the Special Relativity (SR).

In this paper it is presented that in RAF theory there are also two the most intriguing conclusions, regarding to the EMT of gravitational static field. The first one is that the EMT obeys the following properties: 1) the symmetry, 2) the non-negative energy density and 3) zero trace of EMT. The second conclusion is that the gravity force is attractive (in the region $r_{c}<r<\infty, r_{c}=G M / c^{2}$ ) and repulsive (in the region $\left.r_{\text {min }} \leq r<r_{c}, r_{\text {min }}=G M / 2 c^{2}\right)$. At the minimal radius $r_{\text {min }}$, repulsive force is maximal. In that sense, the situation at $r_{c}=G M / c^{2}$ corresponds to the point where the acceleration is equal to zero and free fall velocity is maximal and equal to the speed of the light in vacuum c [23-25]. At the same point the potential energy is equal to zero and the kinetic energy is maximal. Further, at this point the attractive gravitational acceleration (force) is changing into the repulsive one and vice versa. Since at the radius $r_{c}$ the kinetic energy is maximal and the potential energy is zero, the free fall motion continues to the minimal radius $r_{\min }$ where the free fall velocity is equal to zero. At the minimal radius the repulsive acceleration (force) is maximal and repulsive motion is started. At the radius $r_{c}$ the repulsive velocity becomes maximal and equal to the speed of the light in vacuum c. Further, repulsive velocity is decreasing to the zero at radius $r \rightarrow \infty$, or at the maximal radius $r_{\max }<\infty$.

In the property 2) of EMT, the non-negative energy density means that the energy density is positive, but at the radius $r_{c}$ is equal to zero. This is the crucial difference between RAF theory of gravitational static field and field gravity approach where there no zero point of the energy density. Namely, at the radius $r_{c}$ we have the changing from the attraction to the repulsion force and therefore the potential energy must be zero. That is why the energy density of EMT in RAF theory of static gravitational field, at the radius $r_{c}$, should be equal to zero. For all radiuses different from $r_{c}$ energy density of EMT is positive in a static gravitational field as we expected that should be.

The theoretical proof that the positive gravitational force could be the source of dark energy is presented in [48]. The generalized relativistic Hamiltonian in an alpha field is derived in [49]. As the result we obtain linear Dirac's like relativistic Hamiltonian as the function of the field parameters $\alpha$ and $\alpha^{\prime}$. By applying quadratic operation to the linear form of the Hamiltonian we obtain the related nonlinear relativistic Hamiltonian in an alpha field. In the case of the vacuum without any potential field $\left(\alpha=\alpha^{\prime}=1\right)$, the nonlinear Hamiltonian is transformed into the well-known nonlinear form, valid in the Special Relativity.

This paper is organized as follows. In Sec. II, we started with the solution of the field parameters $\alpha$ and $\alpha^{\prime}$ in a general form, as the function of the normalized potential energy $U$ of a particle in the related field. Solution of the field parameters $\alpha$ and $\alpha^{\prime}$ in a gravitational field is considered in Sec. III. Derivation of energy-momentum tensor for gravitational field is pointed out in Sec. IV. The theoretical proofs that RAF theory satisfies required properties of EMT in gravitational static field are presented in Sec. V. Finally, the related conclusion and the reference list are presented in Sec. VI and Sec. VII, respectively.

\section{SOLUTION OF THE FIELd PARAMETERS IN AN ALPHA FIELD}

Relativistic Alpha Field Theory (RAFT) is based on the following two definitions [23]:

Definition 1. An alpha field is a potential field that can be described by two dimensionless (unit less) scalar parameters $\alpha$ and $\alpha^{\prime}$. To this category belong, among the others, electrical and gravitational fields.

Definition 2. Field parameters $\alpha$ and $\alpha^{\prime}$ are described as the scalar dimensionless (unit less) functions of the normalized potential energy $U$ of a particle in an alpha field.

The line element in an alpha field is defined by the nondiagonal form with the Riemannian metrics [34-37]:

$$
\begin{aligned}
d s^{2}= & -\alpha \alpha^{\prime} c^{2} d t^{2}-\kappa\left(\alpha-\alpha^{\prime}\right)_{x} c d t d t-\kappa\left(\alpha-\alpha^{\prime}\right)_{y} c d t d y \\
& -\kappa\left(\alpha-\alpha^{\prime}\right)_{z} c d t d z+d x^{2}+d y^{2}+d z^{2} .
\end{aligned}
$$

The relative velocity of a particle in an alpha field, $v_{\alpha}$, is described as the function of the field parameters $\alpha$ and $\alpha^{\prime}$ [23] $\mathrm{v}_{\alpha}=\mathrm{v}-\frac{\kappa\left(\alpha-\alpha^{\prime}\right) \mathrm{c}}{2}$.

In the previous equation $v$ is a particle velocity in the total vacuum (without any potential field), $c$ is the speed of the light in a vacuum and $\kappa$ is a constant. The relations in (1) and (2) satisfy the well-known condition [26,27,35-37] for the metric tensor of the line element (1)

$$
\sqrt{-\operatorname{det}\left(g_{\mu \nu}\right)}=1, \rightarrow \kappa= \pm 1
$$

Let $\mathrm{d} \tau$ and $\mathrm{dt}$ are differentials of the proper time and coordinate time (respectively) of the moving particle in an alpha field. Further, let $H$ is a transformation factor, as an invariant of an alpha field, and $v_{\alpha}$ is a particle velocity in that field given by (2). For that case, the transformation factor $H$ has the following form

$$
H=\frac{d t}{d \tau}=\left(1-\frac{v_{\alpha}^{2}}{c^{2}}\right)^{-1 / 2}=\left(\alpha \alpha^{\prime}-\frac{v^{2}}{c^{2}}+\frac{\kappa\left(\alpha-\alpha^{\prime}\right) c v}{c^{2}}\right)^{-1 / 2} .
$$

Furthermore, if a particle is moving in a total vacuum (without any potential field), then we have $\alpha=\alpha^{\prime}=1$, and the relation (4) is transformed into the transformation factor $\gamma$ valid in the Special Relativity:

$$
\begin{gathered}
\alpha=\alpha^{\prime}=1, \rightarrow H=\frac{d t}{d \tau}=\left(1-\frac{v^{2}}{c^{2}}\right)^{-1 / 2}=\gamma=\frac{d t}{d t^{\prime}}, \\
\rightarrow \quad d \tau=d t^{\prime} .
\end{gathered}
$$

Let $\mathrm{m}_{0}$ is a rest mass of a particle, $\mathrm{U}$ is a potential energy of a particle in an alpha field, $c$ is the speed of the light in a vacuum and $(i)$ is an imaginary unit. In that case the field 
parameters $\alpha$ and $\alpha^{\prime}$ can be described as dimensionless (unit less) functions of the normalized potential energy $U$ of a particle in an alpha field. There are four solutions for both parameters $\alpha$ and $\alpha^{\prime}$ in an alpha field that can be presented by the following relations [23]:

$$
\begin{aligned}
& f(U)=2 U / m_{0} c^{2}+\left(U / m_{0} c^{2}\right)^{2}, \rightarrow \alpha_{1}=1+i \sqrt{f(U)}, \\
& \alpha_{1}^{\prime}=1-i \sqrt{f(U)}, \quad \alpha_{2}=1-i \sqrt{f(U)}, \alpha_{2}^{\prime}=1+i \sqrt{f(U)}, \\
& \alpha_{3}=-1+i \sqrt{f(U)}, \alpha_{3}^{\prime}=-1-i \sqrt{f(U)}, \\
& \alpha_{4}=-1-i \sqrt{f(U)}, \alpha_{4}^{\prime}=-1+i \sqrt{f(U)} .
\end{aligned}
$$

The four solutions of the field parameter $\alpha$ in (6) can be presented in the form

$$
\begin{gathered}
\alpha_{1,2}=1 \pm i \sqrt{\frac{2 U}{m_{0} c^{2}}+\left(\frac{U}{m_{0} c^{2}}\right)^{2}}, \\
\alpha_{3,4}=-1 \pm i \sqrt{\frac{2 U}{m_{0} c^{2}}+\left(\frac{U}{m_{0} c^{2}}\right)^{2}} .
\end{gathered}
$$

The related four solutions of the field parameter $\alpha^{\prime}$ in (6) can be presented with the following relations

$$
\begin{gathered}
\alpha_{1,2}^{\prime}=1 \mp i \sqrt{\frac{2 U}{m_{0} c^{2}}+\left(\frac{U}{m_{0} c^{2}}\right)^{2}}, \\
\alpha_{3,4}^{\prime}=-1 \mp i \sqrt{\frac{2 U}{m_{0} c^{2}}+\left(\frac{U}{m_{0} c^{2}}\right)^{2}} .
\end{gathered}
$$

Thus, the four solutions of the field parameters $\alpha$ and $\alpha^{\prime}$ can be obtained by the unification of the two parameter structures given by (7) and (8):

$$
\begin{aligned}
& f(U)=2 U / m_{0} c^{2}+\left(U / m_{0} c^{2}\right)^{2}, \rightarrow \\
& \alpha_{1,2}=1 \pm i \sqrt{f(U)}, \alpha_{1,2}^{\prime}=1 \mp i \sqrt{f(U)}, \\
& \alpha_{3,4}=-1 \pm i \sqrt{f(U)}, \alpha_{3,4}^{\prime}=-1 \mp i \sqrt{f(U)} .
\end{aligned}
$$

Further, it is easy to prove that all $\alpha_{i} \alpha_{i}^{\prime}$ pairs from (9) are creating an invariant $\alpha \alpha^{\prime}$

$$
\alpha_{i} \alpha_{i}^{\prime}=\left(1+\frac{U}{m_{0} c^{2}}\right)^{2}=\alpha \alpha^{\prime}, \quad i=1,2,3.4 .
$$

For calculation some of the quantities in an alpha field we often need to know the difference of the field parameters:

$$
\begin{aligned}
& \alpha_{1}-\alpha_{1}^{\prime}=2 i \sqrt{f(U)}, \quad \alpha_{2}-\alpha_{2}^{\prime}=-2 i \sqrt{f(U)}, \\
& \alpha_{3}-\alpha_{3}^{\prime}=2 i \sqrt{f(U)}, \quad \alpha_{4}-\alpha_{4}^{\prime}=-2 i \sqrt{f(U)}, \\
& \left(\alpha_{1}-\alpha_{1}^{\prime}\right)=\left(\alpha_{3}-\alpha_{3}^{\prime}\right), \quad\left(\alpha_{2}-\alpha_{2}^{\prime}\right)=\left(\alpha_{4}-\alpha_{4}^{\prime}\right) .
\end{aligned}
$$

The obtained relations in (9), (10) and (11) are valid generally and for their calculation we only need to know potential energy $U$ of the particle in the related potential field.
Remarks 1. From the equations (9), (10) and (11) we can see that there are three very important properties of the solutions of the field parameters $\alpha$ and $\alpha^{\prime}$ : a) parameters $\alpha$ and $\alpha^{\prime}$ are dimensionless (unitless) field parameters, b) there are four solutions of the field parameters $\alpha$ and $\alpha^{\prime}$ that reminds us to the Dirac's theory [38] and c) the quantity $\alpha \alpha^{\prime}$ is an invariant related to the four solutions of the field parameters $\alpha$ and $\alpha^{\prime}$.

\section{SOLUTION OF THE FIELD PARAMETERS IN GRAVITATIONAL FIELD}

If a particle with the rest mass $\mathrm{m}_{0}$ is in a gravitational field, then the potential energy of the particle in that field $U_{g}$ is described by the well-known relation [2,26-30]

$$
U_{g}=m_{0} V_{g}=m_{0} A_{g 0}=-\frac{m_{0} G M}{r} .
$$

Here $V_{g}=A_{g 0}$ is a scalar potential of the gravitational field, $G$ is the gravitational constant, $M$ is a gravitational mass and $r$ is a gravitational radius. The four solutions of the field parameters $\alpha$ and $\alpha^{\prime}$ for the particle in a gravitational field can be obtained by the substitution of the potential energy $U_{g}$ from (12) into the general relations in (9):

$$
\begin{aligned}
& i \sqrt{f\left(U_{g}\right)}=-\sqrt{2 G M / r c^{2}-\left(G M / r c^{2}\right)^{2}}=-\sqrt{\cdot}, \rightarrow \\
& \alpha_{1}=1-\sqrt{\cdot}, \quad \alpha_{1}^{\prime}=1+\sqrt{\cdot}, \quad \alpha_{2}=\alpha_{1}^{\prime}, \quad \alpha_{2}^{\prime}=\alpha_{1}, \\
& \alpha_{3}=-1-\sqrt{\cdot}, \quad \alpha_{3}^{\prime}=-1+\sqrt{\cdot}, \quad \alpha_{4}=\alpha_{3}^{\prime}, \quad \alpha_{4}^{\prime}=\alpha_{3}, \\
& G M \ll r c^{2}, \rightarrow\left(G M / r c^{2}\right)^{2} \cong 0, \rightarrow i \sqrt{f\left(U_{g}\right)}=-\sqrt{2 G M / r c^{2}} .
\end{aligned}
$$

The first three lines in equations (13) describe a strong gravitational field. If the quadratic term $\left(G M / r c^{2}\right)^{2} \approx 0$ then the field parameters (13) describe a relatively weak gravitational field as we have in our solar system.

The differences of the field parameters $\left(\alpha-\alpha^{\prime}\right)$ for a particle in a gravitational field have the forms:

$$
\begin{aligned}
& \alpha_{1}-\alpha_{1}^{\prime}=-2 \sqrt{\frac{2 G M}{r c^{2}}-\left(\frac{G M}{r c^{2}}\right)^{2}}, \alpha_{3}-\alpha_{3}^{\prime}=\left(\alpha_{1}-\alpha_{1}^{\prime}\right), \\
& \alpha_{2}-\alpha_{2}^{\prime}=2 \sqrt{\frac{2 G M}{r c^{2}}-\left(\frac{G M}{r c^{2}}\right)^{2}}, \alpha_{4}-\alpha_{4}^{\prime}=\left(\alpha_{2}-\alpha_{2}^{\prime}\right) .
\end{aligned}
$$

Remarks 2. In the references [24,25,39] it has been shown that the field parameters (13) and (14) satisfy the Einstein's field equations with a cosmological constant $\Lambda=0$. In the case of a strong static gravitational field [40-44], the quadratic term $\left(G M / r c^{2}\right)^{2}$ in (13) and (14) generates the related energy-momentum tensor $T_{\mu \eta}$ for the static field. For that case, we do not need to add by hand the related energy-momentum tensor $T_{\mu \eta}$ on the right side of the Einstein's field equations.

The second interpretation could be that the quadratic term $\left(G M / r c^{2}\right)^{2}$ generates the cosmological parameter $\Lambda$ as a function of a gravitational radius [45] for $T_{\mu \eta}=0$. It has been 
shown [46] that this solution of $\Lambda$ is valid for both Planck's and cosmological scales. In the case of a relatively weak static gravitational field, like in our solar system, the field parameters (13) satisfy the Einstein's field equations in a vacuum $\left(T_{\mu \eta}=0, \Lambda=0\right)$. The general metrics of the relativistic alpha field theory [34] has been applied to the derivation of dynamic model of nanorobot motion in multipotential field [47].

\section{ENERGY-MOMENTUM TENSOR FOR GRAVITATIONAL FIELD}

The basic problem of this section is to determine the energy-momentum tensors for gravitational field in the Einstein's four-dimensional space-time (4D). In that sense, we started with the general line element $d s^{2}$ given by the relation (1). Following the well-known procedure [2,26-30], this line element can be transformed into the spherical polar coordinates in the nondiagonal form

$$
\begin{aligned}
d s^{2}=- & \alpha \alpha^{\prime} c^{2} d t^{2}-\kappa\left(\alpha-\alpha^{\prime}\right) c d t d r+d r^{2} \\
& +r^{2} d \theta^{2}+r^{2} \sin ^{2} \theta d \phi^{2} .
\end{aligned}
$$

The line element (15) belongs to the well-known form of the Riemann's type line element [34-37]

$$
\begin{aligned}
d s^{2}= & g_{00}\left(d x^{0}\right)^{2}+2 g_{01} d x^{0} d x^{1}+g_{11}\left(d x^{1}\right)^{2} \\
& +g_{22}\left(d x^{2}\right)^{2}+g_{33}\left(d x^{3}\right)^{2} .
\end{aligned}
$$

Comparing the equations (15) and (16) we obtain the coordinates and components of the covariant metric tensor, valid for the line element (15):

$$
\begin{aligned}
& d x^{0}=c d t, \quad d x^{1}=d r, \quad d x^{2}=d \theta, \quad d x^{3}=d \phi, \\
& g_{00}=-\alpha \alpha^{\prime}, \quad g_{01}=g_{10}=\frac{-\kappa\left(\alpha-\alpha^{\prime}\right)}{2}, \\
& g_{11}=1, \quad g_{22}=r^{2}, \quad g_{33}=r^{2} \sin ^{2} \theta .
\end{aligned}
$$

Starting with the line element (15) we employ, for the convenient, the following substitutions:

$v=\alpha \alpha^{\prime}, \quad \lambda=\kappa\left(\alpha^{\prime}-\alpha\right) / 2$.

In that case the nondiagonal line element (15) is transformed into the new relation

$$
\begin{aligned}
d s^{2}=- & v c^{2} d t^{2}+2 \lambda c d t d r+d r^{2} \\
& +r^{2} d \theta^{2}+r^{2} \sin ^{2} \theta d \phi^{2} .
\end{aligned}
$$

Using the coordinate system (17), the related covariant metric tensor $g_{\mu \eta}$ of the line element (19) is presented by the matrix form

$$
\left[g_{\mu \eta}\right]=\left[\begin{array}{cccc}
-v & \lambda & 0 & 0 \\
\lambda & 1 & 0 & 0 \\
0 & 0 & r^{2} & 0 \\
0 & 0 & 0 & r^{2} \sin ^{2} \theta
\end{array}\right] .
$$

This tensor is symmetric and has six non-zero elements as we expected that should be. The contravariant metric tensor $g^{\mu \eta}$ of the nondiagonal line element (19), is derived by inversion of the covariant one (20)

$\left[g^{\mu \eta}\right]=\left[\begin{array}{cccc}-1 /\left(v+\lambda^{2}\right) & \lambda /\left(v+\lambda^{2}\right) & 0 & 0 \\ \lambda /\left(v+\lambda^{2}\right) & v /\left(v+\lambda^{2}\right) & 0 & 0 \\ 0 & 0 & 1 / r^{2} & 0 \\ 0 & 0 & 0 & 1 / r^{2} \sin ^{2} \theta\end{array}\right]$

The determinants of the tensors (20) and (21) are given by the relations:

$$
\begin{aligned}
& \operatorname{det}\left[g_{\mu \eta}\right]=-r^{4}\left(v+\lambda^{2}\right) \sin ^{2} \theta \\
& \operatorname{det}\left[g^{\mu \eta}\right]=-\left(\frac{1}{r^{4}\left(v+\lambda^{2}\right) \sin ^{2} \theta}\right) .
\end{aligned}
$$

Proposition 1. If the gravitational static field is described by the line element (19), then the solution of the Einstein field equations gives the energy momentum tensor $T_{\mu \eta}$ of that field in the following form

$$
\begin{aligned}
T_{\mu \eta}=\left(T_{00}, T_{01}, T_{10}, T_{11}, T_{22}, T_{33}\right) \\
=\left(v,-\lambda,-\lambda,-1, r^{2}, r^{2} \sin ^{2} \theta\right) \frac{(G M)^{2}}{8 \pi G r^{4}} .
\end{aligned}
$$

Here $G$ and $M$ are the gravitational constant and the gravitational mass, respectively.

Proof of the proposition 1. In order to prove of the proposition 1, we can start with the second type of the Christoffel symbols of the metric tensors (20) and (21). These symbols can be calculated by employing the well-known relation $[2,26-30]$

$$
\Gamma_{\eta \mu}^{\gamma}=\frac{g^{\kappa \gamma}}{2}\left[g_{\kappa \eta, \mu}+g_{\kappa \mu, \eta}-g_{\eta \mu, \kappa}\right], \kappa, \gamma, \eta, \mu=0,1,2,3 \text {. }
$$

Thus, employing (19), (20), (21) and (24), we obtain the second type Christoffel symbols of the spherically symmetric non-rotating body:

$\Gamma_{00}^{0}=\left(\dot{v}+2 \lambda \dot{\lambda}+v^{\prime} \lambda\right) / D, \Gamma_{01}^{0}=\Gamma_{10}^{0}=v^{\prime} / D, \Gamma_{11}^{0}=-2 \lambda^{\prime} / D$,

$\Gamma_{22}^{0}=-2 \lambda r / D, \Gamma_{33}^{0}=\left(-2 \lambda r \sin ^{2} \theta\right) / D, \Gamma_{00}^{1}=\left(-\dot{v} \lambda+2 v \dot{\lambda}+v v^{\prime}\right) / D$,

$\Gamma_{01}^{1}=\Gamma_{10}^{1}=-v^{\prime} \lambda / D, \Gamma_{11}^{1}=2 \lambda^{\prime} \lambda / D, \Gamma_{22}^{1}=-2 v r / D$,

$\Gamma_{33}^{1}=\left(-2 v r \sin ^{2} \theta\right) / D, \Gamma_{12}^{2}=\Gamma_{21}^{2}=\frac{1}{r}, \Gamma_{33}^{2}=-\sin \theta \cos \theta, \Gamma_{13}^{3}=\Gamma_{31}^{3}=\frac{1}{r}$, $\Gamma_{23}^{3}=\Gamma_{32}^{3}=\operatorname{ctg} \theta, \quad D=2\left(v+\lambda^{2}\right), \frac{\partial v}{\partial t}=\dot{v}, \frac{\partial v}{\partial r}=v^{\prime}, \frac{\partial \lambda}{\partial t}=\dot{\lambda}, \frac{\partial \lambda}{\partial r}=\lambda^{\prime}$.

For a static field, the Christoffel symbols $\Gamma_{00}^{0}$ and $\Gamma_{00}^{1}$ are reduced to the simplest form: 


$$
\Gamma_{00}^{0}=\frac{v^{\prime} \lambda}{2\left(v+\lambda^{2}\right)}, \Gamma_{00}^{1}=\frac{v v^{\prime}}{2\left(v+\lambda^{2}\right)}, \frac{\partial v}{\partial r}=v^{\prime}, \frac{\partial \lambda}{\partial r}=\lambda^{\prime} .
$$

In a static field, the other Christoffel symbols in (25) are remaining unchanged.

As it is well known, the determinant of the metric tensor of the line element (19) should satisfy the following condition [2,26-30, 35-37]

$$
\sqrt{-\operatorname{det}\left[g_{\mu \eta}\right]}=\sqrt{r^{4}\left(v+\lambda^{2}\right) \sin ^{2} \theta}=1
$$

Including the normalization of the radius, $r=1$, and the angle $\theta=90^{\circ}$ in (27) we obtain the important relations between the parameters $v$ and $\lambda$ :

$$
\begin{aligned}
& v+\lambda^{2}=1, v=1-\lambda^{2}, \\
& v^{\prime}=-2 \lambda \lambda^{\prime}, v^{\prime \prime}=-2\left(\lambda^{\prime 2}+\lambda \lambda^{\prime \prime}\right)
\end{aligned}
$$

Taking into account the relations (28), the Christoffel symbols in (25) and (26) become the only functions of the parameter $\lambda$.

For calculation of the related components of the Riemannian tensor $R_{\beta \eta \mu}^{\kappa}$ and Ricci tensor $R_{\eta \mu}$ of the line element (19) we can employ the following relations [2,26-30]:

$$
\begin{aligned}
& R_{\beta \eta \mu}^{\kappa}=\Gamma_{\beta \mu, \eta}^{\kappa}-\Gamma_{\beta \eta, \mu}^{\kappa}+\Gamma_{\sigma \eta}^{\kappa} \Gamma_{\beta \mu}^{\sigma}-\Gamma_{\sigma \mu}^{\kappa} \Gamma_{\beta \eta}^{\sigma}, \\
& R_{\eta \mu}=R_{\eta \kappa \mu}^{\kappa}=R_{\mu \eta}, \kappa, \beta, \eta, \mu, \sigma=0,1,2,3 .
\end{aligned}
$$

Applying the Christoffel symbols (25) to the relations (29) we obtain the related Ricci tensor for the static field of the line element (19), with the following components:

$$
\begin{aligned}
& R_{00}=-\left(1-\lambda^{2}\right)\left(\lambda^{\prime 2}+\lambda \lambda^{\prime \prime}+\frac{2 \lambda \lambda^{\prime}}{r}\right), \\
& R_{01}=R_{10}=-\lambda\left(\lambda^{\prime 2}+\lambda \lambda^{\prime \prime}+\frac{2 \lambda \lambda^{\prime}}{r}\right), \\
& R_{11}=\left(\lambda^{\prime 2}+\lambda \lambda^{\prime \prime}+\frac{2 \lambda \lambda^{\prime}}{r}\right), R_{22}=2 \lambda \lambda^{\prime} r+\lambda^{2}, \\
& R_{33}=\left(2 \lambda \lambda^{\prime} r+\lambda^{2}\right) \sin ^{2} \theta .
\end{aligned}
$$

The other components of the Ricci tensor are equal to zero. The related Ricci scalar for the static field is determined by the equation

$$
\begin{aligned}
& R=g^{\mu \eta} R_{\mu \eta}, \quad \mu, \eta=0,1,2,3, \rightarrow \\
& R=2\left(\lambda^{\prime 2}+\lambda \lambda^{\prime \prime}+\frac{2 \lambda \lambda^{\prime}}{r}\right)+2\left(\frac{2 \lambda \lambda^{\prime}}{r}+\frac{\lambda^{2}}{r^{2}}\right) .
\end{aligned}
$$

In order to calculate the energy-momentum tensor $T_{\mu \eta}$ for the static field, one should employ Ricci tensor (30), Ricci scalar (31) and the Einstein's field equations [2,26-30] without a cosmological constant $(\Lambda=0)$

$$
R_{\mu \eta}-\frac{1}{2} g_{\mu \eta} R=k T_{\mu \eta}, \quad k=\frac{8 \pi G}{c^{4}}, \quad \mu, \eta=0,1,2,3 .
$$

Here $G$ is the Newton's gravitational constant, $c$ is the speed of the light in a vacuum and $T_{\mu \eta}$ is the energy-momentum tensor. Thus, employing the Einstein's field equations (32) we obtain the following relations for calculation of the components of the energy-momentum tensor $T_{\mu \eta}$ :

$$
\begin{aligned}
& k T_{00}=\left(1-\lambda^{2}\right)\left(\frac{2 \lambda \lambda^{\prime}}{r}+\frac{\lambda^{2}}{r^{2}}\right), k T_{01}=k T_{10}=-\lambda\left(\frac{2 \lambda \lambda^{\prime}}{r}+\frac{\lambda^{2}}{r^{2}}\right), \\
& k T_{11}=(-1)\left(\frac{2 \lambda \lambda^{\prime}}{r}+\frac{\lambda^{2}}{r^{2}}\right), k T_{22}=\left(-r^{2}\right)\left(\lambda^{\prime 2}+\lambda \lambda^{\prime \prime}+\frac{2 \lambda \lambda^{\prime}}{r}\right), \\
& k T_{33}=\left(-r^{2} \sin ^{2} \theta\right)\left(\lambda^{\prime 2}+\lambda \lambda^{\prime \prime}+\frac{2 \lambda \lambda^{\prime}}{r}\right), \quad k=\frac{8 \pi G}{c^{4}} .
\end{aligned}
$$

For calculation of the components of the energy-momentum tensor, $T_{\mu \eta}$, by the relations (33) we should know the parameter $\lambda$ and its derivations $\lambda^{\prime}$ and $\lambda^{\prime \prime}$ for the related static field. Parameter $\lambda$ is defined by (18) as the function of the field parameters $\alpha$ and $\alpha^{\prime}$

$\lambda=\kappa\left(\alpha^{\prime}-\alpha\right) / 2=-\kappa\left(\alpha-\alpha^{\prime}\right) / 2, \quad \kappa= \pm 1$.

Applying the solution of the field parameters $\alpha$ and $\alpha^{\prime}$ in a gravitational field $(13,14)$ to the relation $(34)$ we obtain the two solutions of the parameter $\lambda$ in a gravitational static field

$$
\lambda=\mp \kappa \sqrt{\frac{2 G M}{r c^{2}}-\left(\frac{G M}{r c^{2}}\right)^{2}} .
$$

Now, one can calculate the all components needed for determination of the energy-momentum tensor $T_{\mu \eta}$ in a static gravitational field:

$$
\begin{aligned}
& \lambda^{\prime}=\mp \kappa\left[\frac{G M}{r^{2} c^{2}}-\left(\frac{G M}{r^{2} c^{2}}\right)\left(\frac{G M}{r c^{2}}\right)\right] / \sqrt{\frac{2 G M}{r c^{2}}-\left(\frac{G M}{r c^{2}}\right)^{2},} \\
& \lambda \lambda^{\prime}=\left[-\frac{G M}{r^{2} c^{2}}+\left(\frac{G M}{r^{2} c^{2}}\right)\left(\frac{G M}{r c^{2}}\right)\right], \\
& \frac{2 \lambda \lambda^{\prime}}{r}=2\left[-\frac{G M}{r^{3} c^{2}}+\left(\frac{G M}{r^{2} c^{2}}\right)^{2}\right], \frac{\lambda^{2}}{r^{2}}=\left[\frac{2 G M}{r^{3} c^{2}}-\left(\frac{G M}{r^{2} c^{2}}\right)^{2}\right], \\
& \lambda^{\prime 2}+\lambda \lambda^{\prime \prime}=\left(\lambda \lambda^{\prime}\right)^{\prime}=\frac{2 G M}{r^{3} c^{2}}-3\left(\frac{G M}{r^{2} c^{2}}\right)^{2} .
\end{aligned}
$$

Applying the relations (36) to the equations (31) and (33) we obtain the components of the energy-momentum tensor and Ricci scalar valid for the static gravitational field: 


$$
\begin{aligned}
& k T_{00}=\left(1-\lambda^{2}\right)\left(\frac{G M}{r^{2} c^{2}}\right)^{2}=v\left(\frac{G M}{r^{2} c^{2}}\right)^{2}, \\
& k T_{01}=k T_{10}=-\lambda\left(\frac{G M}{r^{2} c^{2}}\right)^{2}, k=\frac{8 \pi G}{c^{4}}, \\
& k T_{11}=(-1)\left(\frac{G M}{r^{2} c^{2}}\right)^{2}, \quad k T_{22}=r^{2}\left(\frac{G M}{r^{2} c^{2}}\right)^{2} \text {, } \\
& k T_{33}=\left(r^{2} \sin ^{2} \theta\right)\left(\frac{G M}{r^{2} c^{2}}\right)^{2} \text {, } \\
& R=2\left(\lambda^{\prime 2}+\lambda \lambda^{\prime \prime}+\frac{2 \lambda \lambda^{\prime}}{r}\right)+2\left(\frac{2 \lambda \lambda^{\prime}}{r}+\frac{\lambda^{2}}{r^{2}}\right) \\
& =-2\left(\frac{G M}{r^{2} c^{2}}\right)^{2}+2\left(\frac{G M}{r^{2} c^{2}}\right)^{2}=0 .
\end{aligned}
$$

From the previous relations we can see that the Ricci scalar is equal to zero. Finally, included parameter $k$ into the relations (37), we obtain the components of the energy-momentum tensor of the static gravitational field

$$
\begin{aligned}
T_{\mu \eta}= & {\left[T_{00}, T_{01}, T_{10}, T_{11}, T_{22}, T_{33}\right] } \\
& =\left[\nu,-\lambda,-\lambda,-1, r^{2}, r^{2} \sin ^{2} \theta\right] \frac{(G M)^{2}}{8 \pi G r^{4}} .
\end{aligned}
$$

Because the relation (38) is equal to the relation (23), the proof of the proposition 1 is finished.

\section{PROOFS THAT RAF THEORY SATISFIES REQUiRED PROPERTIES OF ENERGY-MOMENTUM TENSOR IN GRAVITATIONAL FIELD}

As it is well known from the quantum relativistic field theories of the other physical interactions, the energy momentum tensor (EMT) of massless boson field obeys the following three crucial conditions [10]: 1) symmetry, $T_{\mu \eta}=T_{\eta \mu} ; 2$ ) positive energy density for static and free field, $T_{00}>0$; and 3) zero trace, $T=0$.

It is very important to prove if the EMT in RAF theory also obeys the mentioned three crucial conditions. In order to prove this, we started with the matrix form of EMT in RAF theory (38)

$$
\left[T_{\mu \eta}\right]=\left[\begin{array}{rrcc}
\nu & -\lambda & 0 & 0 \\
-\lambda & -1 & 0 & 0 \\
0 & 0 & r^{2} & 0 \\
0 & 0 & 0 & r^{2} \sin ^{2} \theta
\end{array}\right] \frac{(G M)^{2}}{8 \pi G r^{4}} .
$$

Following (39) one can see that the matrix in (39) has the symmetric form. This means that EMT in (39) satisfies the first crucial condition: 1) symmetry, $T_{\mu \eta}=T_{\eta \mu}$.

In order to prove the condition of positive energy density for static field, one can start with the element $T_{00}$ of the EMT in (39) and with the relations (28) and (35):

$$
\begin{aligned}
& T_{00}=v \frac{(G M)^{2}}{8 \pi G c^{4}}, \nu=1-\lambda^{2}, \lambda=\mp \kappa \sqrt{\frac{2 G M}{r c^{2}}-\left(\frac{G M}{r c^{2}}\right)^{2}} \\
& v=\left(1-\frac{G M}{r c^{2}}\right)^{2} \rightarrow T_{00}=\left(1-\frac{G M}{r c^{2}}\right)^{\frac{(G M)^{2}}{8 \pi G c^{4}}}
\end{aligned}
$$

where $\kappa= \pm 1$. From the relations in $(40)$ we can see that $T_{00}$ is positive or equal to zero:

$$
r \neq \frac{G M}{c^{2}} \rightarrow T_{00}>0, \quad r=\frac{G M}{c^{2}} \rightarrow T_{00}=0
$$

Thus, one can say that $T_{00}$ can not be negative quantity.

Following the relations (40) and (41) we can see that EMT for gravitational static field satisfies the second crucial condition: 2) positive energy density for static field, $T_{00}>0$ if $r \neq G M / c^{2}$. In the case that $r=G M / c^{2}$, the energy density for static and free field is equal to zero, $T_{00}=0$. Thus, the energy density of EMT for a static gravitational field in RAF theory is non-negative item $T_{00} \geq 0$.

Remarks 3. In RAF theory of the gravitational static field, the situation at $r=G M / c^{2}$ corresponds to the point where the acceleration is equal to zero and free fall velocity is maximal and equal to the speed of the light in vacuum c [23,24,25]. At the same point potential energy is zero and kinetic energy is maximal. Further, at this point negative gravitational force is changing into the positive one and vice versa. This includes possibility that the gravitational force can be both attractive (in the region $r_{c}<r<\infty, r_{c}=G M / c^{2}$ ) and repulsive (in the region $\left.r_{\text {min }} \leq r<r_{c}, r_{\text {min }}=G M / 2 c^{2}\right)$. At the minimal radius $r_{\text {min }}$, repulsive force is maximal.

In order to prove the third crucial condition of EMT: 3 ) the zero trace, $T=0$, we have to calculate the trace of the EMT in (39):

$$
\begin{aligned}
& T=g^{\mu \eta} T_{\mu \eta}=\left[-2\left(\nu+\lambda^{2}\right)+2\right] \frac{(G M)^{2}}{8 \pi G r^{4}}, \\
& \nu+\lambda^{2}=1 \rightarrow T=0 .
\end{aligned}
$$

In the previous relations $g^{\mu \eta}$ is contravariant metric tensor (21) of a gravitational static field and $T_{\mu \eta}$ is the related covariant energy-momentum tensor (39). The relation $v+\lambda^{2}=1$ is derived from the condition that the determinant of the metric tensor of the line element (19) should satisfy the relations (27) and (28).

From the previous consideration we can derive the following conclusion. In RAF theory the energy momentum tensor (EMT) of the gravitational static field obeys the following three crucial conditions: 1 ) symmetry, $T_{\mu \eta}=T_{\eta \mu}$; 2) positive or null energy density for static field, $T_{00} \geq 0$ and 3 ) zero trace, $T=0$. Here the condition 2) can be change into the related new condition: 2) non-negative energy density 
for static field, $T_{00} \geq 0$. In the condition 2) of EMT the non-negative energy density means that the energy density is positive, but at the radius $r_{c}$ is equal to zero. This is the crucial difference between field gravity approach $\left(T_{00}>0\right)$ and RAF theory of gravitational static field ( $\left.T_{00} \geq 0\right)$.

\section{CONCLUSION}

In this paper we consider the properties of the energy-momentum tensor (EMT) in a static gravitational field. This tensor is derived in the Relativistic Alpha Field Theory (RAFT). It is shown that the EMT of gravitational static field obeys the following properties: 1) the symmetry, 2 ) the non-negative energy density and 3) zero trace of EMT. Here the non-negative energy density means that the energy density is positive, but at the radius $r_{c}=G M / c^{2}$ is equal to zero. This radius corresponds to the point where the acceleration is equal to zero because negative acceleration is changing into the positive one and vice versa. This is the crucial difference between RAF theory of gravitational static field and field gravity approach, where energy density is positive and without null point. Further it is shown that the gravity force is attractive (in the region $r_{c}<r<\infty$ ) and repulsive (in the region $r_{\min } \leq r<r_{c}$, where $r_{\min }=G M / 2 c^{2}$ ). At the minimal radius $r_{\text {min }}$, repulsive force is maximal.

\section{ACKNOWLEDGMENTS}

This work is supported by grants (120-1201842-3048) from the National Scientific Foundation of Republic of Croatia.

\section{REFERENCES}

[1] D. Hilbert, Gottingen Nachrichten 4, 21, (1917).

[2] A. Einstein, Ann. D. Phys. 49, 769-822 (1916).

[3] E. Schrödinger, Phys. Zeitschr., 19, 4, (1918).

[4] H. Bauer, Phys. Zeitschr., 19, 163, (1918).

[5] L. D. Landau, E. M. Lifshitz, The Classical Theory of Fields, Pergamon, Oxford, (1971).

[6] A. A. Logunov, M. A. Mestvirishvili, The Relativistic Theory of Gravitational, Mir, Moscow, (1989).

[7] H. Yilmaz, Nuovo Cimento, 107 B, 941, (1992)

[8] S. V. Babak, L. P. Grishchuk, Phys. Rev. D61, 024038, (2000).

[9] N. Straumann, astro-ph/0006423, (2000).

[10] Y. V. Baryshev, Energy-Momentum of the Gravitational Field: Crucial Point for Gravitation Physics and Cosmology, arXiv, October, (2008), www.researchgate.net/publication/1774586.

[11] G. D. Birkhoff, Proc. Nat. Acad. Sci. U.S. 29, 231, (1943).

[12] W. E. Thirring, Ann. of Phys. 16, 96, (1961).

[13] G. Kalman, Phys. Rev. 123, 384, (1961).

[14] R. Feynman, F. Morinigo, W. Wagner, Feynman Lectures on Gravitation, Addison-Wesley Publ. Comp., (1995).

[15] S. Weinberg, Phys. Rev., B 138, 988, (1965).

[16] V. I. Zakharov, Zh. Eksp. Teor. Fiz., 48, 303, (1965).

[17] V. I. Ogievetsky, I. V. Polubarinov, Ann. Phys. 35, 96, (1965).

[18] Yu. V. Baryshev, V. V. Sokolov, Astrophphysics, 21, 361, Thirring, (1984).

[19] V. V. Sokolov, Astrophys. Sp. Sci., 191, 231, (1992).

[20] V. V. Sokolov, Astrophys. Ap. Sp. Sci., 198, 53, (1992).

[21] Yu. V. Baryshev, Proc. of the 1-st Int. Conf. "Crisis in Cosmology", AIP Conf. Proc., 822, 144, (2006).

[22] T. Padmanabhan, gr-gc/0409089, (2004).

[23] B. M. Novakovic, Relativistic alpha field theory - Part I: Determination of Field Parameters. Int. J. New Techn. Res. (IJNTR), 1. 5. ID-IJNTR01050015, 23-30 (2015). https://doi.org/10.31871/IJNTR.1.5.15

[24] B. M. Novakovic, Relativistic alpha field theory-Part II: Does a Gravitational Field Could be Without Singularity? Int. J. New Techn. Res. (IJNTR), 1. 5. ID-IJNTR01050016, 31-38 (2015). https://doi.org/10.31871/IJNTR.1.5.16
[25] B. M. Novakovic, Relativistic alpha field theory-Part III: Does Gravitational Force Becomes Positive if $\left(\mathrm{GM} / \mathrm{rc}^{2}\right)>1$ ? Int. J. New Techn. Res. (IJNTR), 1. 5. ID-IJNTR01050017, 39-47 (2015). https://doi.org/10.31871/IJNTR.1.5.17

[26] A. Einstein, The Meaning of Relativity (Princeton Univ. Press, Princeton, 1955).

[27] C. Sean, Spacetime and Geometry: An introduction to General Relativiry (Amazon.com Books.htm, Hardcover, 2003).

[28] S. Weinberg, Gravitation and Cosmology: Principles and Application of the General Theory of Relativity (Gebundene Ausgabe, RelEspWeinberg.pdf., 1972).

[29] S. W. Hawking, G. F. R. Ellis, The Large Scale Structure of Space-Time (Univ. Press, Cambridge, 1973).

[30] M. Blau, Lecture Notes on General Relativity (A. Einstein Center for Fundamental Physics, Univ. Bern, Bern, 2012, 2014).

[31] B. M. Novakovic, Are Singularity and Dark Energy Consequences of Vacuum Solution of Field Equations?, Int. J. New Techn. Res. (IJNTR), 3, 11. ID-IJNTR03110008, 47-55, (2017). https://doi.org/10.31871/IJNTR.3.11.8

[32] B. M. Novakovic, RAF Theory Extends the Applications of GRT to the Extremely Strong Gravitational Field, Int. J. New Techn. Res. (IJNTR), 3, 11, ID-IJNTR03110009, 56-62, (2017). https://doi.org/10.31871/IJNTR. 3.11.9

[33] B. M. Novakovic, Is Positive Gravitational Force Source of Dark Energy? Int. J. New Techn. Res. (IJNTR), 1. 7. ID-IJNTR01070005, 6-13 (2015). https://doi.org/10.31871/IJNTR.1.7.5

[34] B. M. Novakovic, Int. J. of Comput. Anticip. Syst., IJCAS 27, p. 93 (2014).

[35] S. Gallot, D. Hullin, and D. J. Lafontane, Riemannian Geometry ( Springer-Verlag, Berlin, New York, ed. 3, 2004).

[36] C. T. J. Dodson, and T. Poston, Tensor Geometry. Graduate Texts in Mathematics (Springer-Verlag, Berlin, New York, ed. 2, 1991), p. 130

[37] M. T. Vaughin, Introduction to Mathematical Physics (Wiley-VCH Verlag GmbH \& Co., Weinheim, 2007).

[38] P. A. M. Dirac, Directions in Physics (Wiley, New York, 1978).

[39] B. M. Novakovic, Relativistic alpha field theory, e-Book, Amazon.com, published (2016).

[40] D. H. Perkins, Introduction to High Energy Physics (Cambridge Univ. Press, Cambridge, 2000).

[41] D. Sherman, et al., Nat. Phys. 11, 1-5 (2015).

[42] J. Steinhauer, Nat. Phys. 10, 864-869 (2014).

[43] M. Meckel, et al. Nat. Phys. 10, 594 - 600 (2014).

[44] R. Ding, et al. Phys. Rev. D 92, (015008) (2015).

[45] B. M. Novakovic, D. Novakovic, and A. Novakovic. AIP-CP 718, p.133 (2004). https://doi.org/10.1063/1.1787318

[46] B. M. Novakovic, D. Novakovic, and A. Novakovic, Cosmological Constant Problem Solution Valid for Both Planck's and Cosmological Scales. AIP-CP 839, 144-156, (2006). https://doi.org/10.1063/1.2216624

[47] B. M. Novakovic, Strojarstvo 53 (2), 103-111 (2011).

[48] B. M. Novakovic, Generalized Relativistic Hamiltonian in an Alpha Field. AIP-CP 1303, 141-148 (2010). https://doi.org/10.1063/1.3527149

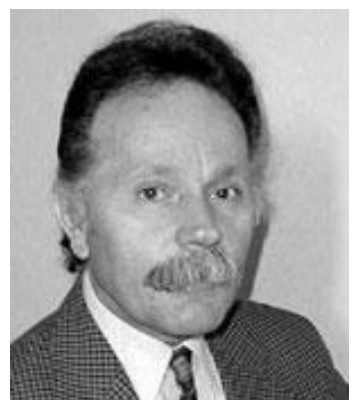

Branko Novakovic is a Professor emeritus at FSB - University of Zagreb, Croatia. Prof. Novakovic received his $\mathrm{PhD}$ from the University of Zagreb in 1978. His research of interest includes physics, control systems, robotics, neural networks, and fuzzy control. He is author of three books; Relativistic Alpha Field Theory (RAFT), e-book (2016), Control Methods in Robotics, Flexible Manufacturing Systems and Processes (1990), Control Systems (1985) and the first co-author of the book Artificial Neural Networks (1998). He has published over 230 research papers in his research of interest. 